\title{
Timolipoma gigante assintomático na infância: relato de caso
}

\author{
Asymptomatic giant thymolipoma in childhood: a case report
}

Carlos Humberto Vicuña ${ }^{1}$, Walter Roriz ${ }^{2}$, Emanuel Torquato ${ }^{2}$, Alberto R Gonçalves ${ }^{3}$, Paulo Faria ${ }^{4}$ Edson Toscano ${ }^{5}$

\section{Resumo}

Reportamos o caso de uma tumoração volumosa localizada no mediastino anterior superior em uma criança do sexo feminino, negra, de 2 anos de idade. A paciente estava assintomática enão apresentava doenças associadas. Foi realizada avaliação pré-operatória usando a tomografia computadorizada e a ressonância nuclear magnética do tórax, que sugeriram o diagnóstico de timolipoma. Este tumor foi tratado cirurgicamente por uma toracotomia bilateral com secção transversa do esterno (incisão à Clamshell). 0 diagnóstico foi confirmado pela histopatologia. O timolipoma é um tumor raro do mediastino anterior que pode apresentar-se como uma grande massa em um paciente assintomático bem como associado com miastenia gravis, esclerose lateral amiotrófica, aplasia medular, hipogamaglobulinemia e liquen plano, entidades estas presentes principalmente nos adultos.

Palavras-chave: timolipoma; neoplasias do timo; lipoma; neoplasias do mediastino; toracotomia; diagnóstico; cirurgia.

\section{Abstract}

We report a case of a huge mass located at the anterior - superior mediastinum in a 2 - year - old black girl. The patient had no symptoms or any other associated disease. Preoperative examination was performed using chest CT scan and chest M RI, and sugested the diagnosis of thymolipoma before surgery. This tumor was succesfully resected by an anterior bilateral thoracotomy with sternotomy (Clamshell incision). The diagnosis was confirmed histologically. Thymolipoma is a rare benign mediastinal tumor that can be presented as very large tumor in an asymptomatic patient, or it can be associated with myasthenia gravis, amyotrophic lateral sclerosis, red cell aplasia, hypogammaglobulinemia and lichen planus, especially in adults.

Key words thymolipoma; thymusneoplasms, lipoma; mediastinal neoplasms, thoracotomy; diagnostic; surgery.

\footnotetext{
${ }^{1}$ Cirurgião Pediátrico, titular do Serviço de Cirurgia Pediátrica do IN CA. Enviar orrespondência para C.H .V. Serviço deC irurgia Pediátrica, PraçaC ruz Vermelha 23, Centro; 20231-092 Rio deJaneiro, RJ - Brasil. E-mail: vicuna@inca.org.br

${ }^{2}$ CirurgiõesTorácicos, titulares do Serviço deC irurgia Torácica do IN CA:

${ }^{3}$ Cirurgião Pediátrico, Chefe do Serviço de Cirurgia Pediátrica do IN CA;

4Patologista, titular do Serviço deAnatomia Patológica do IN CA;

${ }^{5}$ Cirurgião Torácico, Chefe do Serviço de CirurgiaTorácica do IN CA.

Recebido em maio de 2001.
} 


\section{INTRODUÇÃO}

O timolipoma é um tumor raro do mediastino anterior que pode atingir todas as faixas etárias; apresenta-se com crescimento lento, atinge grandes volumes e manifesta-se com poucos sintomas respiratórios. Pode estar associado com a miastenia gravis (M G) e com a esclerose lateral amiotrófica (ELA) principalmente nos adultos. 0 diagnóstico pode ser deduzido com os estudos de imagem, principalmente a ressonância nuclear magnética do tórax. 0 tratamento é cirúrgico e consiste da ressecção completa do tumor havendo poucos casos descritos na literatura médica.

\section{RELATO DE CASO}

C riança negra, do sexo feminino com dois anos de idade, com relato de tratamento para tuberculose por seis meses devido à opacidade em hemitórax direito, sem melhora. Paciente apresentava-se assintomática e ao exame físico observava-se abaulamento da face anterior do hemitórax direito e murmúrio vesicular abolido nessa região. 0 estudo radiológico do tórax $(R X)$ revelou opacificação quase completa do hemitórax direito e discreto desvio do mediastino para a esquerda com deformidade do gradil costal ipsilateral (Figura 1). A tomografia computadorizada (TC) de

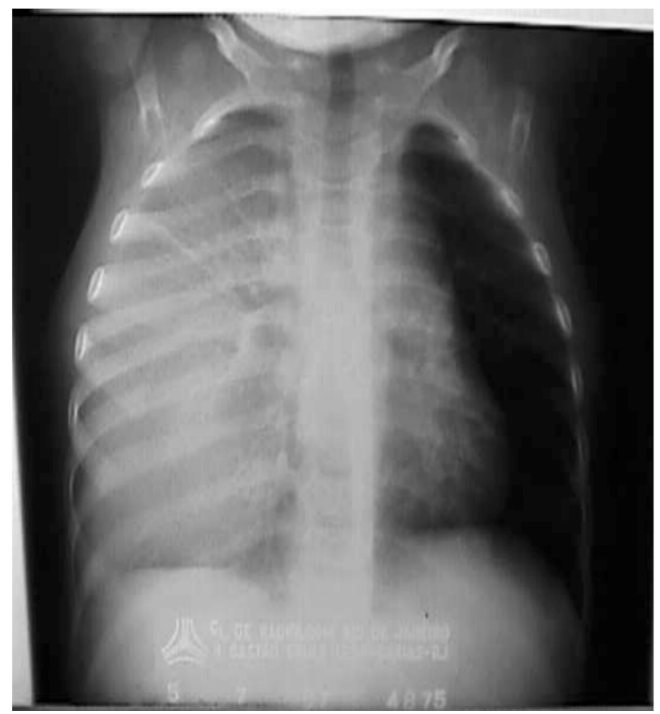

Figura 1. Raio X detórax exibindo opacificação quase completa do hemi-tórax direito, discreto desvio de mediastino para esquerda e deformidade do gradil costal direito. tórax revelou massa sólida, homogênea, sem sinais de calcificação, de bordos convexos, bem delimitada ocupando o mediastino antero-superior e estendendo-se para 0 hemitórax direito, ocupando-o em grande parte (porção anterior) e determinando desvio posterior do brônquio-fonte direito e atelectasia parcial do pulmão correspondente.

O bservava-se ainda pequena área de condensação no segmento ápico-posterior do lobo superior do pulmão esquerdo (Figura 2). A ressonância magnética ( $R M)$ do tórax demonstrou tumoração com áreas de sinal de alta intensidade interligado com áreas de densidade intermediária em T 1 e T 2 (Figura 3). O s achados radiológicos sugeriram lesão expansiva de origem tímica. O s exames laboratoriais e a broncoscopia realizadas anteriormente não ofereceram informações adicionais. Como os exames de imagem não demonstravam sinais de irressecabilidade foi indicada toracotomia para diagnóstico e ressecção do tumor do mediastino anterior. D evido à grande extensão da tumoração optamos pela realização de uma toracotomia bilateral anterior. Após preparo préoperatório planejado em conjunto com a anestesiologia, a cirurgia e o intensivista pediátrico, o paciente foi submetido a uma toracotomia anterior bilateral (incisão à Clamshell) com secção transversa do esterno, que permitiu abordagem completa da tumoração. $\mathrm{N}$ a exploração da cavidade observamos uma tumoração extensa, pediculada, originada no mediastino anterior eocupando grande parte do hemitórax direito,

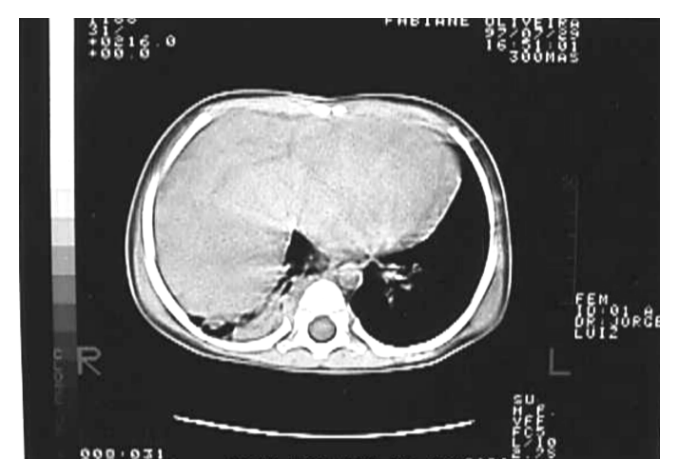

Figura 2. Tomografia computadorizada de tórax: massa sólida homogênea, sem sinais de calcificação, de bordos convexos, bem delimitada ocupando o mediastino antero-superior e extendendo-se para o hemi-tórax direito, ocupando-o em grande parte (porção anterior), determinando desvio posterior do brônquio fonte direito e atelectasia parcial do pulmão direito correspondente. 
comprimindo e deslocando o pulmão direito posteriormente e superiormente, porém sem aderência ou invasão de orgãos adjacentes. Após identificação e preservação do nervo frênico direito, procedemos à exérese cirúrgica da tumoração através do isolamento e secção do seu pedículo vascular. Após remoção do tumor houve expansão completa do pulmão direito. A cavidade pleural foi drenada bilateralmente, a parede costal foi suturada com dois pontos de fio de aço número 3 no esterno e com pontos de fio sintético absorvível no plano costal.

No pós-operatório observamos reexpansão pulmonar completa, sem intercorrências e a alta hospitalar ocorreu no 50 dia de pós-operatório. Atualmente a paciente encontra-se assintomatica e no 350 mês de seguimento.

A macroscopia da lesão mostrava uma superfície externa lisa, limitada por cápsula delgada e transparente pardo-clara e levementelobulada(Figura 4a; b). A superfície de corte era pardo-esbranquiçada com finas traves de permeio delimitando lóbulos, firmeelástica euntuosa, medindo aproximadamente $15 \times 8 \times 7,5 \mathrm{~cm}$, pesando $440 \mathrm{gr}$, dependente do mediastino anterior. 0 estudo histopatológico per-operatório de congelação evidenciou tecido adiposo maduro, sugestivo de neoplasia lipomatosa benigna. 0 exame anátomo patológico mostrou tratar-se de timolipoma benigno.

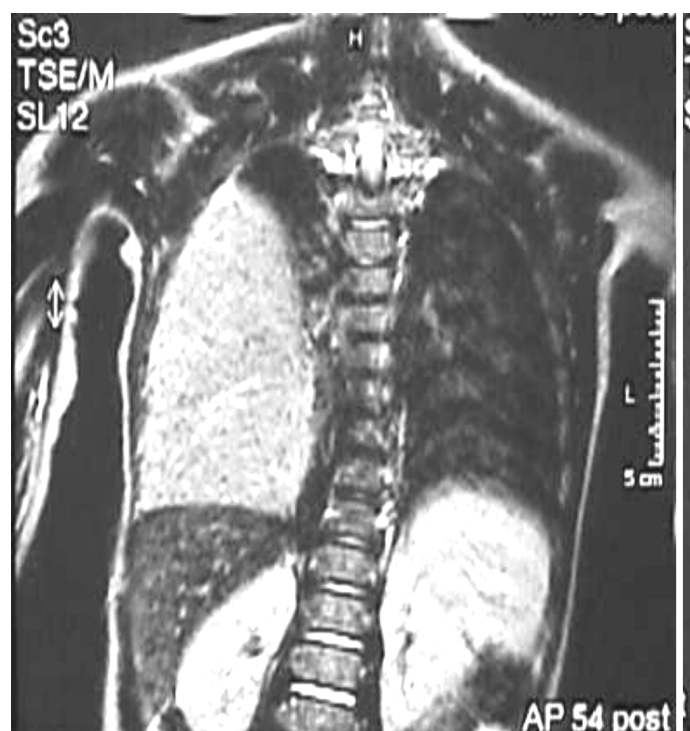

Figura 3. Ressonância magnética (RM) do tórax: tumoração de mediastino anterior com áreas de sinal de alta intensidade interligado com áreas de densidade intermediaria em T 1 eT 2.

\section{DISCUSSÃO}

0 primeiro caso foi reportado por Lange em 1916 como lipoma do timo. ${ }^{1}$ Posteriormente, $\mathrm{H}$ all em 1948 propôs o termo timolipoma. ${ }^{2} 0$ timolipoma é um tumor Acomete uma faixa etária ampla, desde préescolares até adultos, com leve predomínio em pacientes abaixo de 40 anos. ${ }^{3-5}$ Tem crescimento lento, podendo atingir a totalidade do hemitorax sem ocasionar sintomas compressivos. ${ }^{6} \mathrm{~N}$ os adultos pode apresentar-se concomitantemente com miastenia gravis, esclerose lateral amiotrófica, bem como com aplasia medular, hipogamaglobulinemia e liquen plano. ${ }^{4,5,7,8}$ Embora seja de apresentação rara, 0 timolipoma deve ser considerado para 0 diagnóstico diferencial das massas do mediastino anterior. ${ }^{9-12} \mathrm{~A}$ hiperplasia maciça verdadeira do timo é uma entidade rara que pode ser incluida no diagnóstico diferencial do timolipoma. ${ }^{13}$ A patogênese está relacionada com alterações degenerativas dos corpúsculos de $\mathrm{H}$ assal e com infiltração lipomatosa na glândula. ${ }^{6}$ À histopatologia, apresenta-se como lóbulos de tecido lipomatoso maduro com ilhotas de células tímicas contendo células epiteliais, linfócitos e corpúsculos de $\mathrm{H}$ assal. ${ }^{14}$ As células epiteliais podem ser imunorreativas a citoqueratina. ${ }^{15}$ benigno raro do mediastino antero-superior. ${ }^{3}$








Figura 4a. Toracotomia anterior bilateral ( incisão à clamshell ) com secção do esterno;

Áreas de calcificação e degeneração cística também podem estar presentes. ${ }^{6,14,16}$ 0 timofibrolipoma foi descrito como uma variante deste tumor. ${ }^{17}$ As manifestações clínicas podem ser mínimas, e a maioria dos pacientes é assintomática. Eventualmente, pode apresentar-se associado a quadros asmáticos, principalmente em crianças e adultos jovens, ${ }^{10}$ e a infecção de vias respiratórias altas, dor torácica e massa cervical. ${ }^{16} 0$ diagnóstico pode ser sugerido pela radiografia simples de tórax, que revela um tumor do mediastino anterior ocupando parcial ou quase totalmente 0 hemitórax e comprimindo o parênquima pulmonar. ${ }^{10,18} \mathrm{~A}$ tomografia computadorizada e a ressonância nuclear magnética do tórax confirmarão a lesão do mediastino anterior e poderão demonstrar o tecido adiposo infiltrando a glândula tímica. ${ }^{18-20} 0$ tratamento cirúrgico é a única opção terapêutica, podendo ser feito por meio de esternotomia mediana, toracotomia, ou por acessos combinados; no caso aqui descrito foi feita toracotomia anterior bilateral com esternotomia (incisão à Clamshell), via esta que nos propocionou um amplo acesso cirúrgico à lesão. A excisão completa do tumor é facilitada pela dissecção romba em uma tumoração sem aderências aos tecidos vizinhos, tomando-se cuidado de identificar, isolar e ligar os vasos mamários internos, identificar e preservar os nervos frênicos. ${ }^{10,12,19,21}$

A abordagem anestesiológica éfundamental e deve ser planejada entre cirurgião, anestesista e intensivista, para otimizar 0

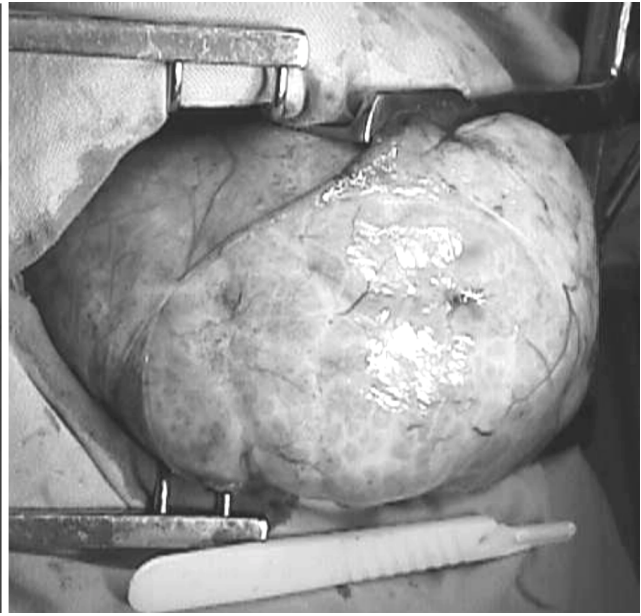

Figura 4b. Exposi ção completa da lesão, cuja superfícieexterna era lisa, limitada por cápsula delgada e transparente pardoclara, e levemente lobulada.

manejo destes pacientes, pois estes tumores volumosos constituem-se num verdadiero desafio pelo grau de compressão brônquica que a lesão exerce num paciente anestesiado. ${ }^{10}$

\section{REFERÊNCIAS BIBLIOGRÁFICAS}

1. LangeL. U ber ein lipom desthymus. Zentralbl Allg Pathol 1916;27:97-101.

2. H all GFM . A case of thymolipoma with observationson a possiblerelationship to intrathoracic lipomata. Br J Surg 1948;36:321-4.

3. Sirpal YM, Chadha SK, Banarjee AK. Thymolipomaararemediastinal tumour-report of two cases and review of literature. Indian J Cancer 1995;32(1):23-6.

4. Takamori $\mathrm{S}, \mathrm{H}$ ayashi A, TayamaK, O htsukaS, $\mathrm{H}$ iraki H, Shirouzu K. Thymolipoma associated with myasthenia gravis. Scand $C$ ardiovasc J 1997;31(4):241-2.

5. Verbist J, Sel R, Van Eyken P, Van Deun J, Schroe H. M yasthenia gravis associated with thymolipoma: a case report. Acta Chir Belg 1997;97(2):97-9.

6. Toyama T, M izuno T, M asaoka A, Shibata K, Yamakawa Y, N iwa H, et al. Pathogenesis of thymolipoma: report of threecases. SurgToday 1995;25(1):86-8.

7. Yoshida H, Sato M, Saito Y, O no S, Sugita M , Kobayashi S, et al. An experience of surgery for thymolipomain apatient with amyotrophic lateral sclerosis. Kyobu G eka 1995;48(13):1092-5.

8. M CM anus KG, Allen M S, Trastek VF, D eschamps $C$, C rotty TB, Pairolero PC. Lipothymoma with red cell aplasia, 
hypogammaglobulinemia and lichen planus. Ann Thorac Surg 1994;58:1534.

9. M entzelT, Kriegsmann J, Kosmehl $H$, Katenkamp D. Ectopic hamartomatous thymoma. Case report with special reference to differential diagnosis. Pathologe 1995;16(5):359-63.

10. Gregory AK, Connery CP, RestaFlarer F, D avis $J E$, Semel $L, H$ olgersen LO . A case of massive thymolipoma. J Pediatr Surg 1997;32(12): 1780-2.

11. M ountney J, Survana SK, Brown PW G, ThorpeJAC. Inflamatory pseudotumor of the lung mimicking thymoma. Eur J Cardiothorac Surg 1997;12(5):801-3.

12. Rosenberg JC. N eoplasms of themediastinum. In: D evitaVT, H ellman S, Rosenberg SA, editors. $C$ ancer: principles and practice of oncology. 4th ed. Philadelphia: JB Lippincott; 1993.

13. Woywodt A, Verhaart S, Kiss A . M assivetrue thymic hyperplasia. Eur J Pediatr Surg 1999;9:331-3.

14. H ayashi A, Takamori S, TayamaK, M itsuoka M , O htsukaS, A oyamaY, et al. Thymolipoma: clinical and pathological features-report of three cases and review of literature. Kurume $M$ ed J 1997;44(2):141-6.
15. H ull M T, Warfel KA, Kotylo P, Goheen M P, Brown JW. Proliferating thymolipoma: ultrastructural, immunohistochemical, and flowcytometric study. U Itrastruct Pathol 1995; 19(1):75-81.

16. M oran CA, Rosado-de-Christenson M L, Suster S. Thymolipoma: clinicopathologic review of 33 cases. M od Pathol 1995;8(7):741-4.

17. Moran CA, Zeren $H$, Koss $M N$. Thymofibrolipoma: a histologic variant of thymolipoma. Arch Pathol Lab Med 1994;118(3):281-2.

18. Rosado-de-Christenson $M L$, Pugatch RD, M oran CA, G al obardesJ. Thymolipoma: analysis of 27 cases. Radiology 1994;193(1):121-6.

19. Kitano Y, Yokomori K, O hkura M , KataokaT, $\mathrm{N}$ arita M , TakemuraT. Giant thymolipoma in a child. J Pediatr Surg 1993;28(12):1622-5.

20. $\mathrm{M}$ atsudaira $\mathrm{N}, \mathrm{H}$ irano $\mathrm{H}$, Itou S, $\mathrm{M}$ atsuuraK, $K$ anou $M, O$ gawa $T$. M R imaging of thymolipoma. M agn Reson Imaging 1994;12(6):959-61.

21. Breek JK, Vallaeys JH, Rutsaert RR. Simultaneous presentation of a thyrolipoma and a thymolipoma in a young man. Eur J Surg 1997;163(12):941-3. 